\title{
A two-photon probe based on naphthalimide-styrene fluorophore for the in vivo tracking of cellular senescence
}

\author{
Beatriz Lozano-Torres, ${ }^{1,2,3,4}$ Juan F Blandez, ${ }^{1,2,4}$ Irene Galiana, ${ }^{1,2,3,4}$ José A Lopez-Dominguez, ${ }^{5}$ Miguel \\ Rovira, ${ }^{5}$ Marta Paez-Ribes, ${ }^{6}$ Estela González-Gualda, ${ }^{6}$ Daniel Muñoz-Espín, ${ }^{6}$ Manuel Serrano, ${ }^{5,7}$ Félix \\ Sancenón ${ }^{1,2,3,4}$ and Ramón Martínez-Máñez ${ }^{1,2,3,4 *}$
}

${ }^{1}$ Instituto Interuniversitario de Investigación de Reconocimiento Molecular y Desarrollo Tecnológico (IDM), Universitat Politècnica de València-Universitat de València, Camí de Vera S/N, Valencia, 46022 Spain.

${ }^{2}$ Unidad Mixta UPV-CIPF de Investigación en Mecanismos de Enfermedades y Nanomedicina, Universitat Politècnica de València, Centro de Investigación Príncipe Felipe, C/ Eduardo Primo Yúfera 3, Spain Valencia, 46012, Spain.

${ }^{3}$ CIBER de Bioingeniería, Biomateriales y Nanomedicina (CIBER-BBN), Av. Monforte de Lemos, 3-5. Pabellón 11. Planta 0, Madrid, 28029, Spain

${ }^{4}$ Unidad Mixta de Investigación en Nanomedicina y Sensores. Universitat Politècnica de València, IIS La Fe, Av. Fernando Abril Martorell, 10, Torre A $7^{\mathrm{a}}$ planta, Valencia, 46026, Spain.

${ }^{5}$ Institute for Research in Biomedicine (IRB Barcelona). Barcelona Institute of Science and Technology (BIST), Carrer de Baldiri Reixac, 10, Barcelona, 08028, Spain.

${ }^{6}$ CRUK Cancer Centre Early Detection Programme, Department of Oncology, University of Cambridge, Hutchison/MRC Research Centre, Box 197, Cambridge, CB2 0XZ, UK

${ }^{7}$ Catalan Institution for Research and Advanced Studies (ICREA), Passeig Lluís Companys 23, 08010Barcelona, Spain

*Correspondence: $\underline{\text { rmaez@qim.upv.es }}$

Cellular senescence, two-photon probe, senescence-inducing chemotherapy, renal fibrosis, diagnosis, in vivo validation.

\begin{abstract}
Cellular senescence is a state of stable cell cycle arrest that can negatively affect the regenerative capacities of tissues, and can contribute to inflammation and the progression of various ageing-related diseases. Advances in the in vivo detection of cellular senescence are still crucial to monitor the action of senolytic drugs and also to assess the early onset or accumulation of senescent cells. Here, we describe a naphthalimide-styrene-based probe (HeckGal) for the detection of cellular senescence both in vitro and in vivo. HeckGal is hydrolyzed by the increased lysosomal $\beta$-galactosidase activity of senescent cells, resulting in fluorescence emission. The probe was validated in vitro using normal human fibroblasts and various cancer cell lines undergoing senescence induced by different stress stimuli. Remarkably, HeckGal was also validated in vivo in an orthotopic breast cancer mouse model treated with senescence-inducing chemotherapy, and in a renal fibrosis mouse model. In all cases, HeckGal allowed the unambiguous detection of senescence in vitro as well as in tissues and tumors in vivo. This work is expected to provide a potential technology for senescence detection in aged or damaged tissues.
\end{abstract}

Cellular senescence is a biological process occurring in response to stress or damage and whose main role is to trigger tissue repair and to prevent the proliferation of stressed or damaged cells. ${ }^{1}$ Senescence triggered by excessive proliferation is known as "replicative senescence", 2 but senescence can also be triggered through diverse procedures such as activation of oncogenes, inhibition of tumor suppressor genes, accumulation of DNA damage, presence of reactive oxygen species (ROS), or nucleolar stresses among others. This type of senescence is known as stressinduced premature senescence (SIPS). ${ }^{3}$ Senescence has a relevant physiological role during development and promotes tissue regeneration in response to circumstantial damage, but the inefficient elimination of senescent cells during aging or upon persistent damage can produce inflammation, fibrosis, tissue aging, tumorigenesis, and metastasis. ${ }^{1,4-6}$

Evidence is accumulating that the selective elimination of senescent cells ameliorates a wide variety of aging-associated diseases, reverts long-term degenerative processes, and extends both lifespan and healthspan in mice. ${ }^{7,8}$ Inspired by these findings, there is a growing interest in developing drugs capable to induce apoptosis preferentially in senescent cells. In fact, senotherapies (treatments with senolytic or senomorphic drugs) are a new strat- egy to prevent cell-autonomous and non-cell-autonomous effects of senescent cells. ${ }^{9}$ Senolytic drugs kill senescent cells preferentially over non-senescent cells; whereas senomorphic drugs reduce the secretion of pro-inflammatory and pro-fibrotic factors by senescent cells but without killing them. ${ }^{10}$ Such drugs would contribute to the therapeutic treatment of senescence-associated diseases and may stimulate the long-term idea that rejuvenation might be possible. ${ }^{6}$ A related important issue in the field of senotherapy is the development of new highly selective and sensitive tools to detect cellular senescence. ${ }^{11}$ These probes are expected to play an essential role in the detection of senescent cells in aged or damaged tissues, help in the discrimination between senolytic (selectively killing senescent cells) and senomorphic (selectively suppressing SASP) drugs or monitor the action of senotherapeutics in multiple age-related disorders. ${ }^{10}$

Some of the most important markers of senescent cells are senescence-associated heterochromatic foci (SAHF), ${ }^{12,13}$ activation of tumor suppressors and cell cycle inhibitors (e.g. p53, p16INK4a and $\mathrm{p} 21),{ }^{14-17}$ the overexpression of anti-apoptotic proteins (e.g. BCLs), ${ }^{18}$ the absence of proliferative markers (Ki67), ${ }^{19}$ the loss of important chromatin structural proteins (Lamin B1, HMGB1, and HGMB2), ${ }^{20}$ a senescence-associated secretory phenotype 
$(\mathrm{SASP})^{21}$ and the presence of high levels of lysosomal $\beta$ galactosidase $(\beta-\mathrm{Gal})$ activity, known as senescence-associated $\beta$ galactosidase, $(\mathrm{SA}-\beta \mathrm{Gal}){ }^{22}$ Monitoring $\beta$-Gal activity using chromo-fluorogenic molecular-based probes represents a simple and accurate manner to track senescence in most of the cases and several $\beta$-Gal probes are commercially available. However, most of these probes cannot be applied to in vivo models. ${ }^{10}$ For example, fluorescein-di-( $\beta$-D-galactopyranoside) (FDG) requires the use of chloroquine in order to increase lysosomal $\mathrm{pH}^{23,24} 4$ methylumbelliferyl $\beta$-D-galactopyranoside (MUG) is not permeable to cells, ${ }^{25}$ and 5-bromo-4-chloro-3-indolyl- $\beta$-Dgalactopyranoside $(\mathrm{X}-\mathrm{Gal})^{26}$ is toxic and therefore cells need to be previously fixed. These drawbacks have boosted the interest in developing molecular sensors for the monitoring of $\beta-G a l$ activity that could work on live cells and tissues. More recently, ((E)-2-(2(6-hydroxy-2,3-dihydro-1H-xanthen-4-yl)vinyl)-3,3-dimethyl-1propyl-3H-indol-1-ium), known as Spider-Gal, has gained importance as SA- $\beta$ Gal kit detection, especially for flow cytometry since the fluorophore is covalently anchored to the cell after hydrolysis. But this could be a problem when applying in vivo and cause long-term toxicity. ${ }^{27}$ Most of the described probes are based on classical one-photon fluorophores linked to the anomeric carbon of $\beta$-galactose. In one-photon probes, the biological target is only detected by an intensity-responsive fluorescent signal, which can be interfered with the excitation and emission efficiency, probe concentration, and surrounding conditions. ${ }^{28,29}$ As an alternative, the design of molecular probes using two-photon fluorophores has attracted great attention in the last years due to their improved three-dimensional spatial localization, prolonged observation time, increased imaging depth, minimized fluorescence background and light scattering, and lower tissue injury. ${ }^{30} \mathrm{Be}-$ sides, one typical inaccuracy when developing SA- $\beta$-Gal probes is their validation in biological models not directly related to senescence. In most cases, the method used in previous in vitro studies are based on lac $\mathrm{Z}$ gene transfection and in vivo using mouse models in which tumors are labelled with avidin- $\beta-\mathrm{Gal}^{31,32}$ or transfected with the pCMV-lacZ plasmid, ${ }^{33}$ that results in high levels of cytosolic $\beta$-Gal expression, ${ }^{34-41}$ which is unrelated to the lysosomal human $\beta$-Gal, encoded by GLB1 gene, naturally overexpressed in senescent cells. As a consequence, there are still a very limited number of selective two-photon fluorescent probes for the detection of bona-fide cellular senescence in vivo models. Specifically, SG1 was the first two-photon ratiometric probe to detect senescent cells in vitro, ${ }^{42}$ and $\mathrm{AHGa}$ was the first twophoton probe to detect cellular senescence in vivo. ${ }^{43}$

Taking into account our interest in the development of fluorogenic sensors, ${ }^{44-48}$ we report herein the synthesis and characterization of a new two-photon naphthalimide-styrene probe (HeckGal in Figure 1A) for in vivo detection of senescence. The HeckGal probe consists of a naphthalimide-styrene fluorophore (Heck in Figure 1A) covalently linked to an acetylated $\beta$-galactose through the anomeric carbon. HeckGal is poorly emissive, whereas a sudden revival of the emission is observed in the presence of lysosomal $\beta$-Gal activity. The probe is tested in vitro in human cancer cell lines, including SK-Mel-103 and A549 cells, a breast murine cancer line (4T1), and in a human BJ fibroblast cell line, undergoing senescence by different triggers. The probe is also tested in vivo in $\mathrm{BALB} / \mathrm{cByJ}$ female mice bearing $4 \mathrm{~T} 1$ breast cancer tumors treated with senescence-inducing chemotherapy, and in a model of renal fibrosis induced by treatment with folic acid in $\mathrm{C} 57 \mathrm{BL} / 6 \mathrm{~J}$ male mice.

\section{EXPERIMENTAL SECTION}

Materials. All chemical reagents were purchased from SigmaAldrich while anhydrous solvents and phosphate-buffered saline (PBS, 0.01M) were purchased from Sharlab S.L., and used with- out further purification. Palbociclib was purchased from Selleckchem and Dulbecco's Modified Eagle Medium (DMEM) and fetal bovine serum (FBS) were purchased from Gibco. Flat-bottomclear 96 well plates were purchased from Promega. Highresolution mass spectrometry (HRMS) and the data was recorded with a TRIPLETOF T5600 (ABSciex, USA) spectrometer. 1H and 13C NMR spectra were collected on a Bruker FT-NMR Avance 400 (Ettlingen, Germany) spectrometer at 300K, using TMS as an internal standard. HPLC measures were obtained by a Walters 1525 binary HPLC pump and spectra were recorded by a Walters 2998 photodiode array at $260 \mathrm{~nm}$. Fluorescence spectra were recorded by a JASCO FP-8500 fluorescence spectrophotometer, Luminescence was collected in a VICTOR Multilabel Plate Reader (Perkin Elmer). Confocal fluorescence images were taken on a Leica TCS SP8 AOBS and two-photon images were acquired by using a multiphoton Olympus FV1000MPE confocal microscope. Images were analyzed using the Image J software. SKMel-103 (human melanoma) cancer cell line and 4T1 (breast cancer cells) were acquired from the American Type Culture Collection (ATCC). BALB/CBY female mice were purchased from Charles River laboratories, France

Hydrolysis reaction. The hydrolysis reaction of the HeckGal probe by $\beta$-Gal enzyme was analyzed by fluorescence spectroscopy and by HPLC-UV techniques. For this purpose, $2 \mu$ l of human $\beta$-Gal enzyme was added to PBS (pH 7)-DMSO (0.01\%) solutions of HeckGal $\left(10^{-5} \mathrm{M}\right)$, and the emission spectrum at $552 \mathrm{~nm}$ was recorded with time (Figure S7a). After 15 min, HeckGal was completely hydrolyzed and the emission band of the product closely correlated with the emission intensity of pure Heck fluorophore solution. Furthermore, in the same reaction condition HPLC-UV studies (Figure S7b) corroborated these results. Timeconversion plots of HeckGal and its reaction intermediated (Heck and $\beta$-Gal) were determined by analyzing reaction aliquots by reversed-phase liquid chromatography using a KromasilC18 column as the stationary phase, eluting under isocratic conditions $0.8 \mathrm{~mL} / \mathrm{min}$ (87.4:12.5:0.1 vol\% $\left.\mathrm{H}_{2} \mathrm{O} / \mathrm{CH}_{3} \mathrm{CN} / \mathrm{CH}_{3} \mathrm{COOH}\right)$ and using photodiode array detector. Retention time (Rt) for Heck was $18.17 \mathrm{~min}$, while Rt for HeckGal was $8.55 \mathrm{~min}$ and $4.60 \mathrm{~min}$ for human $\beta$-Gal enzyme.

Cell lines. SK-Mel-103 (human melanoma) cancer cells and 4T1 (mouse breast cancer cells) were obtained from ATCC. Cells were maintained in DMEM, supplemented with $10 \%$ FBS, and incubated in $20 \% \mathrm{O}_{2}$ and $5 \% \mathrm{CO}_{2}$ at $37^{\circ} \mathrm{C}$. Cells were routinely tested for mycoplasma contamination using the mycoplasma tissue culture NI (MTC-NI) Rapid Detection System (Gen-Probe). For senescence induction, cells were supplemented for 2 weeks with media containing $5 \mu \mathrm{M}$ palbociclib.

In vitro viability assays. SK-Mel-103 (human melanoma) cancer and 4T1 (mouse breast cancer) cells were used for cell viability assays. Cells were maintained in DMEM supplemented with $10 \%$ FBS and incubated in $20 \% \mathrm{O}_{2}$ and $5 \% \mathrm{CO}_{2}$ at $37^{\circ} \mathrm{C}$. For senescence induction, cells were supplemented with DMEM media containing $5 \mu \mathrm{M}$ palbociclib for 2 weeks. Control and senescent cells were placed in flat-bottom-clear 96 well plates at a density of 6,000 and 4,000 cells per well, respectively. The following day cells were treated with serial dilutions of HeckGal or Heck. Viability was assessed $48 \mathrm{~h}$ later with CellTiter-GLO Luminescent Cell Viability Assay. Raw data were obtained by measuring luminescence in a VICTOR Multilabel Plate Reader (Pelkin Elmer).

Mouse models. Balb/cByJ mice were maintained at the Spanish Research Centre Principe Felipe (CIPF) in accordance with the 


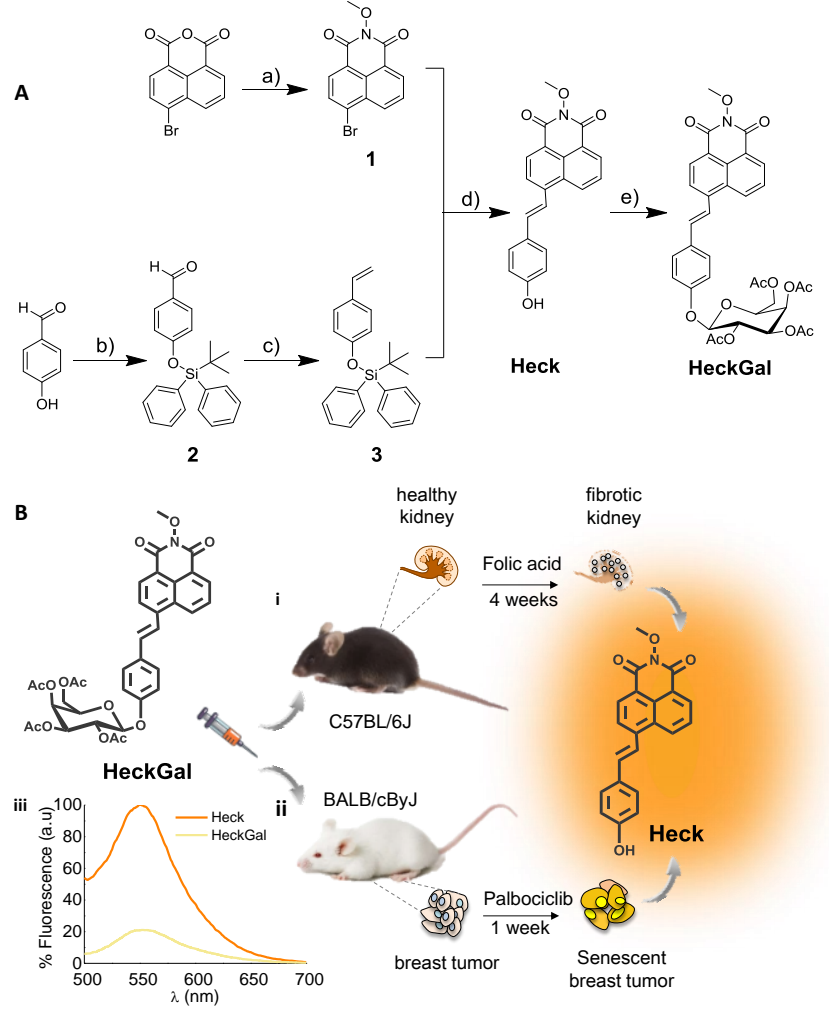

Figure 1. Synthesis of probe and mechanism of action in mice. (A) Synthetic route used for the preparation of the probe. (B) Schematic representation of the application of the probe in two in vivo models of senescence: i) kidney fibrotic $\mathrm{C} 57 \mathrm{BL} / 6 \mathrm{~J}$ male mice induced by treatment with folic acid and ii) BALB/cByJ female mice bearing $4 \mathrm{~T} 1$ breast cancer tumours treated with senescence-inducing chemotherapy. iii) Fluorescence emission spectra $\left(\lambda_{\mathrm{ex}}=488 \mathrm{~nm}\right)$ of HeckGal (yellow) and Heck fluorophore (orange) in aqueous solutions ( $\mathrm{pH} 7)$-DMSO $(0.01 \%)$.

recommendations of the Federation of European Laboratory Animal Science Associations (FELASA). Breast 4T1 tumors were established by using 4T1 cells. Cells were routinely cultured in DMEM supplemented with 10\% FBS and penicillin-streptomycin. In order to generate breast tumors, cells were trypsinised, counted with a LUNA ${ }^{\mathrm{TM}}$ Automated Cell Counter, and injected subcutaneously in the left breast of 28- to 34-week-old BALB/cByJ female mice at a concentration of $0.5 \times 10^{6}$ cells in a volume of 100 $\mu 1$. Tumor volume was measured every two days with a caliper and calculated as $\mathrm{V}=\left(\mathrm{a} \mathrm{x} \mathrm{b}^{2}\right) / 2$ where $\mathrm{a}$ is the longer and $\mathrm{b}$ is the shorter of two perpendicular diameters. Palbociclib or vehicle was administered by daily oral gavage for 7 days at $100 \mathrm{mg} / \mathrm{kg}$ dissolved in $50 \mathrm{mM}$ sodium lactate, $\mathrm{pH} 5$ in order to induce senescence. Then, Heck-Gal was intraperitoneally (i.p.) administered at a concentration of $6 \mathrm{mg} / \mathrm{ml}$ in DMEM (5\% DMSO) in a volume of $200 \mu \mathrm{l}$. Mice were sacrificed 2 hours later by $\mathrm{CO}_{2}$ exposure in a Euthanasia Chamber, and tumors and organs (lung, liver, kidney, or spleen) were immediately removed. Tumors and organs were analyzed immediately after harvesting. Heck was detected using an excitation wavelength of $500 \mathrm{~nm}$ and an emission wavelength of $540 \mathrm{~nm}$. Fluorescence images were taken on an IVIS spectrum imaging system and analyzed by using the Living Imaging software from Caliper Life Sciences. On the other hand, two monthsold C57BL/6J male mice were maintained at the Institut de Recerca Biomèdica (IRB). All animal procedures were carried out in compliance with the regulations of the Animal Care and Use Ethical Committee of the Barcelona Science Park (CEEA-PCB) and the Catalan Government under the recommendations of the FELASA. In order to generate renal fibrosis, mice were i.p injected with a single dose of either $250 \mathrm{mg} / \mathrm{kg}$ of folic acid or vehicle. 34 days after treatment, the animals were administered either with a single i.p dose of Heck-Gal $(13.33 \mathrm{mg} / \mathrm{ml} 200 \mu \mathrm{l})$ in DMSO $1 \%$-Corn oil or with vehicle. Animals were euthanized 5 hours later by $\mathrm{CO}_{2}$ exposure in a Euthanasia Chamber and the kidneys were excised for observation with an IVIS imager (Perkin Elmer).

\section{Preparation of mouse tumour slices for imaging experiments}

Tumours from Balb/cByJ mice ortothopically injected with 4T1 cells treated or not treated with palbociclib were excised and cut in half. They were pasted onto a petri dish exposing a tumor surface as smooth as possible. The slices were incubated with a $10 \mathrm{mM}$ solution of HeckGal for $2 \mathrm{~h}$ at $37^{\circ} \mathrm{C}$ in a dry incubator, and then washed three times with PBS, and observed under twophoton confocal microscope (OLYMPUS FV1000MPE). The images were acquired at different penetration depths $(\lambda$ ex $=820$ $\mathrm{nm})$.

\section{RESULTS AND DISCUSSION}

Synthesis, Characterization, Spectroscopic Features, and Mechanism.

HeckGal probe was synthesized following the synthetic procedure shown in Figure 1A. Naphthalimide 1 was obtained by the reaction between 4-bromo-1,8-naphthalic anhydride and methoxylamine in refluxing dioxane. In parallel, the hydroxyl group of 4hydroxybenzaldehyde was protected with tbutylchlorodiphenylsilane (TBDPSCl) yielding compound $\mathbf{2}$, in which the aldehyde was converted into a double bond using a Wittig reaction resulting in compound $\mathbf{3}$. A Heck cross-coupling reaction between $\mathbf{1}$ and $\mathbf{3}$ yielded Heck fluorophore. Finally, Heck was consecutively reacted with $\mathrm{NaOH}$, in order to remove the phenolic proton, and with 2,3,4,6-tetra-O-acetyl- $\alpha$-Dgalactopyranosyl bromide ( $\mathrm{Gal}$ ) yielding the HeckGal probe. The final probe and intermediate compounds were fully characterized by ${ }^{1} \mathrm{H}-\mathrm{NMR},{ }^{13} \mathrm{C}-\mathrm{NMR}$, and HRMS (Figure S1-S5). PBS (pH 7)DMSO $(0.01 \%)$ solutions of Heck fluorophore $\left(10^{-5} \mathrm{M}\right)$ presented an intense emission band centered at $550 \mathrm{~nm}\left(\Phi_{\text {Heck }}=0.875\right)$ when excited at $488 \mathrm{~nm}$ (Figure 1B, iii)). In contrast, excitation at $488 \mathrm{~nm}$ of PBS (pH 7)-DMSO (0.01\%) solutions of HeckGal resulted in a weak broad emission $\left(\Phi_{\text {HeckGal }}=0.074\right)$ (Figure $1 \mathrm{~B}$, iii). The low emission intensity of HeckGal, when compared to that of Heck, is ascribed to a photoinduced electron transfer process from the galactose unit to the excited fluorophore. It was also assessed that the emission intensity of Heck remained unchanged in the 4-9 $\mathrm{pH}$ range (Figure S6). After assessing the photophysical properties, time-dependent fluorescent measurements in PBS $(\mathrm{pH}$ 7)-DMSO (0.01\%) solutions of HeckGal in the presence of $\beta$-Gal were carried out (Figure S7, A). Progressive enhancement of the emission at $550 \mathrm{~nm}$ was observed due to the generation of free Heck produced by the enzyme-induced hydrolysis of the Oglycosidic bond in HeckGal. The reaction was also analyzed by HPLC (Figure S7, B) which showed the progressive vanishing of the HeckGal peak (at ca. $8.5 \mathrm{~min}$ ) with the subsequent appearance of Heck signal at ca. $8.2 \mathrm{~min}$.

HeckGal displays several advantages when compared with the recently reported AHGa probe. HeckGal presents a more extended conjugated framework which is reflected in a marked increase, of almost $100 \mathrm{~nm}$, in the two-photon excitation wavelength. This increase in excitation wavelength might allow greater tissue penetrability, less photo-toxicity, and reduced light scattering. Moreover, the molecule generated after HeckGal hydrolysis with $\beta$-gal enzyme (i.e. the Heck fluorophore) shows a remarkable higher quantum yield of 0.875 , making the HeckGal probe more suitable for the differentiation between senescent from non-senescent cells with high basal levels of the $\beta$-Gal enzyme. Besides, a comparative table of HeckGal and other cell senescence probes published 


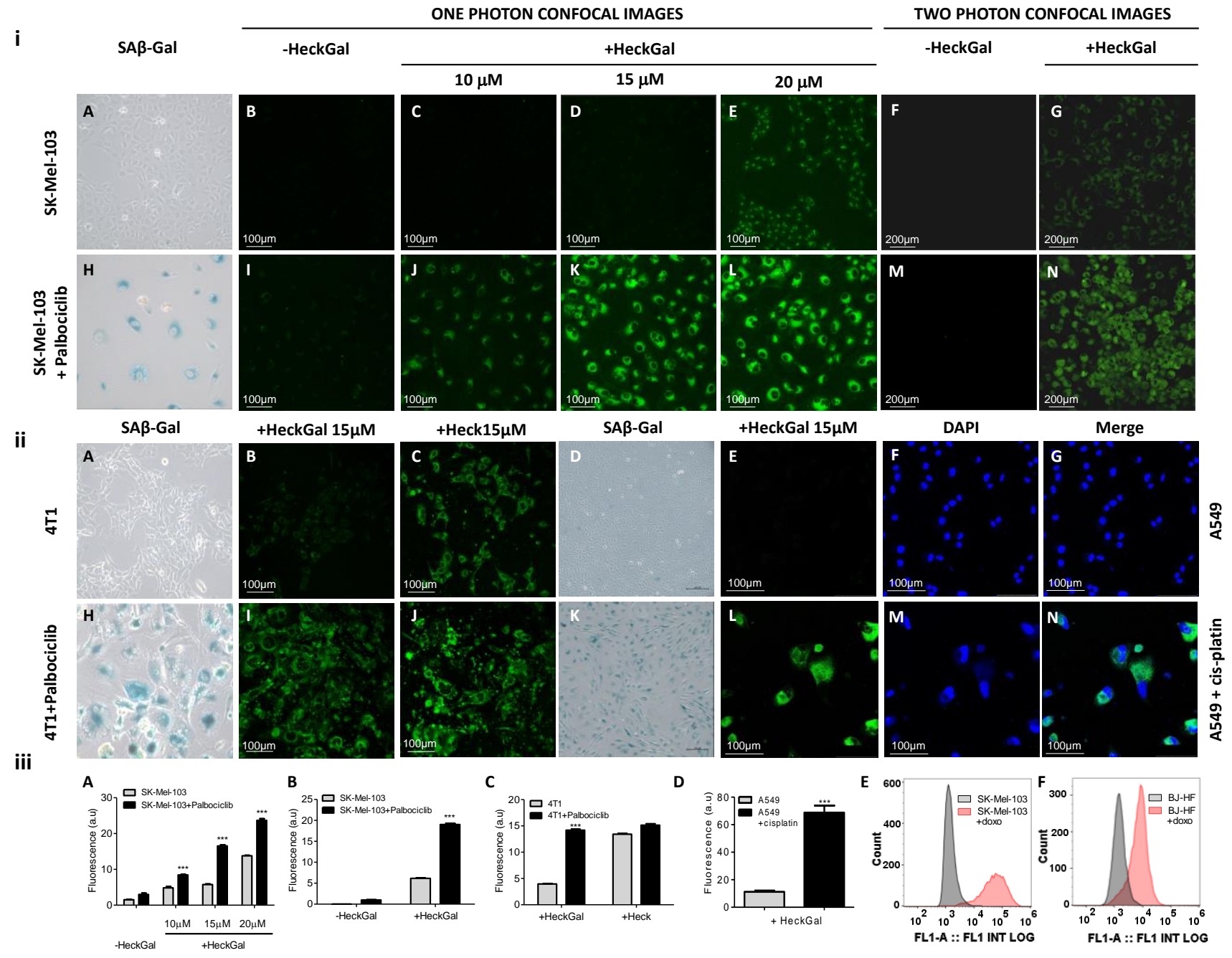

Figure 2. The probe enables the detection of senescence in various cell lines regardless of the induction method.

(i) Senescence induction was assessed by SA $\beta$-Gal staining in non-treated (A) and palbociclib-treated cells(H). Note that senescent SK-Mel-103 cells present the typical blue staining. Confocal images of SK-Mel-103 and SK-Mel-103 treated with palbociclib. One photon confocal images (B-E, I-L) of control SK-Mel-103 (B-E) in the absence (B) or presence of 10, 15, and $20 \mu \mathrm{M}$ of HeckGal probe (C, D, E respectively) and SK-Mel-103 treated with palbociclib (I-L) in the absence (I) or presence of 10,15 and $20 \mu \mathrm{M}$ of HeckGal probe (J, K, L respectively). Two-photon microscopy images (F, G, M, N) of nontreated (F, G) and (M, N) palbociclib-treated (senescent) SK-Mel-103 cells in the absence (F, M) and presence (G, N) of $10 \mu \mathrm{M}$ of HeckGal probe. Cells were incubated with HeckGal in DMEM (10\% FBS, 0.1\% DMSO) in $20 \% \mathrm{O}_{2}$ and $5 \% \mathrm{CO}_{2}$ at $37^{\circ} \mathrm{C}$ for $2 \mathrm{~h}$, and then one photon images were acquired by using a confocal microscope (Leica TCS SP8 AOBS) and two-photon images were acquired by using a multiphoton confocal microscope (OLYMPUS FV1000MPE). (ii) SA -Gal staining of non-treated (A) and palbociclib-treated 4T1 cells (H). Note that senescent $4 \mathrm{~T} 1$ cells present the typical blue staining. Confocal images (B, C, I, J)) of control 4T1 (B, C) in presence of $15 \mu \mathrm{M}$ of HeckGal probe (A) or $15 \mu \mathrm{M}$ of Heck (B) and 4T1 treated with palbociclib (I, $\mathrm{J}$ ) in presence of $15 \mu \mathrm{M}$ of HeckGal probe (I) or $15 \mu \mathrm{M}$ of Heck (J). SA $\beta$-Gal staining of non-treated (D) and cisplatin-treated A549 cells(K). Note that senescent A549 cells present the typical blue staining. Confocal microscopy images of non-treated (E-F) and cisplatin-treated A549 cells (L-N), exposed to the HeckGal probe. Cells were incubated with HeckGal $(15 \mu \mathrm{M})$ in DMEM $+10 \% \mathrm{FBS}$ in $20 \% \mathrm{O}_{2}$ and $5 \% \mathrm{CO}_{2}$ at $37^{\circ} \mathrm{C}$ for $2 \mathrm{~h}$, and images were acquired by using a confocal microscope (excitation at $488 \mathrm{~nm}$ ). (iii) Quantification of the fluorescence emission intensity relative to the cell surface of control and palbociclib-treated SK-Mel-103 cells incubated with HeckGal visualized with one photon confocal imaging (A) and two-photon confocal imaging (B). Quantification of the fluorescence emission intensity relative to the cell surface of control and palbociclib-treated 4T1 cells incubated with HeckGal or Heck visualized with one photon confocal imaging (C). Quantification of the fluorescence emission intensity relative to the cell surface of control and cisplatin-treated A549 cells incubated with HeckGal visualized with one photon confocal imaging (D). Error bars represent SEM (n=3) (E) FluorescenceActivated Cell Sorting (FACS) analysis for control Sk-Mel-103 (grey) human melanoma cells and doxorubicin-treated SK-Mel-103 (red) cells after treatment with HeckGal. (F) FACS analysis for control BJ (grey) human fibroblast cells and doxorubicin-treated BJ (red) cells after treatment with HeckGal. Both cell lines were treated with $250 \mathrm{nM}$ doxorubicin for 24 hours in order to induce cellular senescence, or with DMSO as vehicle. 14 days later, upon complete development of the senescent phenotype, cells were incubated with $7 \mu \mathrm{M}$ HeckGal for 2 hours, detached from the plates, and washed twice with PBS. HeckGal fluorescence was subsequently evaluated by a Sony SA3800 Spectral Analyzer.

in the last three years is shown in the Supporting Information (Table S1).

\section{In Vitro Validation of HeckGal probe}

To study the cellular toxicity after prolonged exposure to the HeckGal probe, human melanoma SK-Mel-103 and murine breast cancer 4T1 cells were used in cell viability assays and the results showed that after $48 \mathrm{~h}$ neither Heck nor HeckGal were toxic for SK-Mel-103 or 4T1 cells, in both senescence and nonsenescence states, at concentrations of up to $100 \mu \mathrm{M}$ (Figure S8). Once proven the probe's biocompatibility, the preferential activation of HeckGal in senescent cells in vitro was assessed in senescent SK-Mel-103, 4T1, A549 (human lung carcinoma), and BJ (human fibroblast) cell lines. Senescence was induced in SK-Mel103 and $4 \mathrm{~T} 1$ cells by treatment with $5 \mu \mathrm{M}$ palbociclib, a well- 

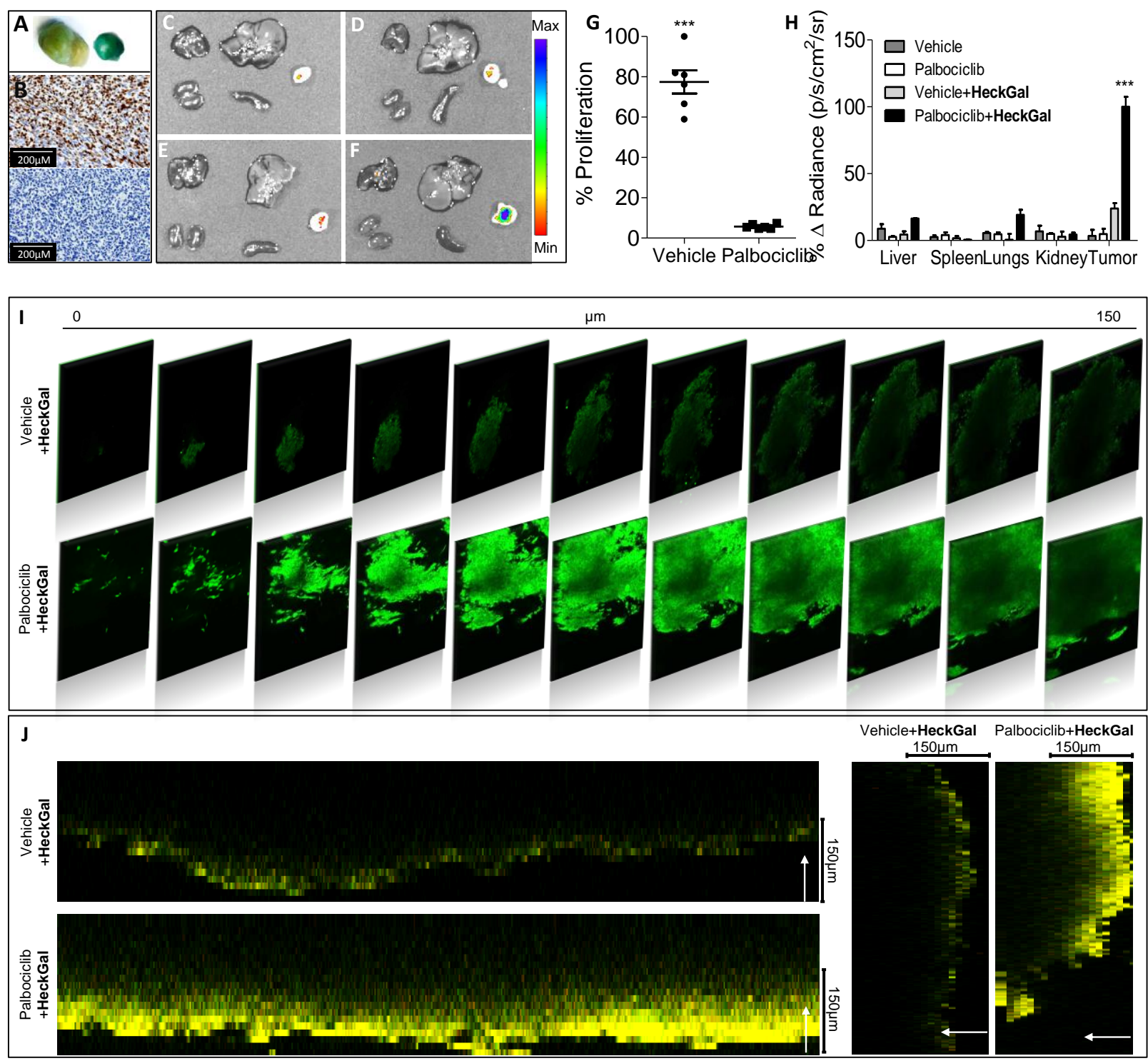

Figure 3. HeckGal probe enables the detection of senescence in different disease models of senescence.

(A) Representative images of tumours stained for the SA- $\beta$-Gal assay. Tumours from vehicle (left) and palbociclib-treated mice (right). (B) Immunohistochemical detection of the proliferation marker Ki67 in paraffin sections of tumors from vehicle (top) and palbociclib-treated mice (bottom). (C-F) IVIS images of organs and tumours from BALB/cByJ female mice bearing 4T1 breast cancer cells. From left to right and from top to bottom: lungs, liver, tumour, kidney, and spleen. (C) Vehicle mice. (D) Vehicle mice treated with $(13.33 \mathrm{mg} / \mathrm{ml} \mathrm{100 \mu l).} \mathrm{(E)} \mathrm{Mice} \mathrm{treated} \mathrm{with} \mathrm{palbociclib} \mathrm{for} 1$ week. (F) Palbociclib-treated mice injected with HeckGal $(13.33 \mathrm{mg} / \mathrm{ml} 100 \mu \mathrm{l})$. Mice were sacrificed two hours post-HeckGal treatment. (G) Quantification of Ki67 signal in paraffin sections of tumours from vehicle (top) and palbociclib-treated mice (bottom). Error bars represent s.d. (H) Quantification of average radiance intensity from organs and tumours showed in images C,D,E, and F. Error bars represent SEM ( $\mathrm{n}=3$ for each condition). (I) Two-photon fluorescence depth images of HeckGal in tumour tissue slices from vehicle (up) and palbociclib-treated mice (down). The slices were incubated with HeckGal (10 mM) for $2 \mathrm{~h}$ at $37^{\circ} \mathrm{C}$ in a dry incubator. The images were acquired at different penetration depths $\left(\lambda_{\mathrm{ex}}=820 \mathrm{~nm}\right)$. (J) 3D representation of images shown in Figure $3 \mathrm{I}$ demonstrating the greater penetrability of HeckGal in tumour tissue slices from palbociclib-treated mice (down) compared to tumour tissue slices from vehicle (up).

known specific CDK4/6 inhibitor, ${ }^{49}$ for two weeks. After palbociclib treatment, the cell morphology changed, presenting an enlarged and flattened appearance typical of cellular senescence. Cellular senescence was assessed by SA- $\beta$-Gal activity assay (Figure 2i (A and $\mathrm{H})$ and 2ii (A and $\mathrm{H})$. Next, control and senescent SK-Mel-103 cells were seeded in flat-bottom-clear 96 wells plates and incubated with 10,15 , and $20 \mu \mathrm{M}$ solutions of HeckGal in DMEM (0.1\% DMSO) for $2 \mathrm{~h}$ in the case of one-photon studies. In the case of two-photon studies, cells were seeded in $\mathrm{p} 6$ wells plates and incubated with a $10 \mu \mathrm{M}$ solution of the probe. Cells were imaged by confocal microscopy using an excitation wavelength of $488 \mathrm{~nm}$, and by two-photon confocal microscopy using a $950 \mathrm{~nm}$ excitation wavelength. Control (Figure 2i (B and F)) and senescent (Figure 2i (I and M)) SK-Mel-103 cells did not show significant background signal before incubation with HeckGal, especially in two-photon studies (Compare I and M in Figure 2i). Non-senescent SK-Mel-103 cells showed weak emission in the presence of increasing concentrations $(10,15$, and 20 $\mu \mathrm{M})$ of the HeckGal probe (Figure 2i (C, D, E, and G)), while palbociclib-treated SK-Mel-103 displayed an intense fluorescent signal, increased for higher HeckGal concentrations (Figure 2i (J, $\mathrm{K}, \mathrm{L}$, and N)). The fluorescent signal in the cells is attributed to the hydrolysis of HeckGal into Heck fluorophore that occurred preferably in senescent cells, which present an increased $\beta$ - 
galactosidase activity. Moreover, the emission spectrum of Heck, obtained after two-photon excitation (Figure S9), corresponds to that obtained in a fluorimeter when using one-photon $488 \mathrm{~nm}$ excitation wavelength (Figure 1 iii). Fluorescence quantification from the confocal images associated with each treatment showed a fluorescence enhancement (ca. 2.9 fold) in palbociclib-treated SK-Mel-103 incubated with $15 \mu \mathrm{M}$ of the probe in one-photon confocal images (Figure 2iii A) and ca. 3.1 fold for cells incubated with $10 \mu \mathrm{M}$ of the probe in two-photon images (Figure 2iii B). Moreover, the ability of HeckGal to detect senescent 4T1 cells was also confirmed. Non-treated and palbociclib-treated (senescent) $4 \mathrm{~T} 1$ cells were incubated with $15 \mu \mathrm{M}$ solutions of HeckGal or Heck in DMEM $(0.1 \%$ DMSO) for $2 \mathrm{~h}$. Figure $2 \mathrm{ii}$ shows that control 4T1 cells treated with HeckGal (Figure 2ii B) showed a minimal fluorescence when compared to senescent 4T1 cells (Figure 2ii I) in the same conditions (3.6-fold enhancement, Figure 2iii C). This marked difference was not observed when control and senescent 4T1 cells were treated with Heck (Figure 2ii C and J), demonstrating the selectivity of HeckGal to detect cellular senescence. The versatility of the HeckGal probe was also validated in other cell lines where senescence was induced with different chemotherapy. Thus, human lung adenocarcinoma (A549) cells were treated with cisplatin $15 \mu \mathrm{M}$ for 3 weeks. Further incubation with HeckGal resulted in an enhanced fluorescence (ca. 6.1 fold, see Figure 2iii D for quantification of images) in cisplatin-treated A549 cells when compared with non-treated A549 cells (Figure 2ii, E and L). Finally, co-staining with typical staining kits did not affect the Heck fluorescence signal or hydrolysis of HeckGal (Figure S10). The use of the HeckGal probe was also assessed by Fluorescence-Activated Cell Sorting (FACS) (Figure 2iii E and F) For these studies, control SK-Mel-103 cells and BJ human fibroblasts (grey) were exposed to $250 \mathrm{nM}$ doxorubicin for $24 \mathrm{~h}$ to induce cellular senescence (red). On day 14, control and senescent cells from both cell lines were treated with $7 \mu \mathrm{M}$ solutions of HeckGal for $2 \mathrm{~h}$, detached from the plates, and fluorescence was subsequently evaluated through FACS. The studies demonstrated that HeckGal can distinguish between control and senescent cell populations in doxorubicin-induced SK-Mel-103 and BJ human fibroblasts.

\section{In Vivo Validation of HeckGal probe}

Encouraged by the ability of HeckGal to detect cellular senescence in vitro, we took a step forward and studied the potential of the HeckGal probe to detect cellular senescence in vivo in two different disease models of senescence: (i) BALB/cByJ female mice bearing $4 \mathrm{~T} 1$ breast cancer tumors treated with palbociclib and (ii) C57BL/6J male mice with renal fibrosis induced by treatment with folic acid (FA). BALB/cByJ female mice were orthotopically injected in the mammary fat pad with $4 \mathrm{~T} 1$ cells $(0.5$ $\mathrm{x} 10^{6}$ cell/mouse) in order to generate breast tumors. Seven days later, palbociclib was administered daily by oral gavage to arrest tumor growth and induce cellular senescence. One week after, palbociclib treatment was started, $100 \mu \mathrm{l}$ of HeckGal was injected intraperitoneally (i.p.) at a concentration of $13.3 \mathrm{mg} / \mathrm{ml}$, and mice were sacrificed $3 \mathrm{~h}$ after treatment. Different organs (i.e. lungs, liver, kidney, and spleen) and tumors were harvested. Cellular senescence in palbociclib-treated tumors was assessed by SA$\beta-$ Gal staining (Figure 3A). The reduction of Ki67, a proliferative marker, observed by immunohistochemistry (IHC) was also indicative of cellular senescence in palbociclib-treated tumors (Figure $3 \mathrm{~B}$ ). Figure $3 \mathrm{G}$ shows the quantification of the Ki67 signal. Ex vivo IVIS images demonstrated that no fluorescent signal was observed in control animals, neither in tumors nor in lungs, liver, kidney, or spleen (Figure 3C, 3D, 3E and 3F), either in the presnce or absence of HeckGal. Tumors of mice treated with palbociclib in the absence of HeckGal were used to monitor tissue auto-fluorescence and displayed a weak emission. In contrast tumors from mice previously treated with palbociclib and i.p. injected with HeckGal showed a strong emission signal in IVIS images (Figure 3F). Quantification of the average radiance intensity from organs and tumors was determined for each condition (Figure $3 \mathrm{H}$ ). An emission enhancement of ca. 4.6 fold was observed in tumors treated with palbociclib when compared to control tumors. These results demonstrate that HeckGal is a potent tool to visualize senescence in a breast cancer tumor model treated with senescence-inducing therapy. Moreover, to evaluate Heck-Gal penetrability and their ability for two-photon imaging of senescent cells in the depth of tissues, fluorescence intensities of tumour slices from vehicle, and palbociclib-treated mice at different depths were measured by a Z-scan model (Fig. 3I and $3 \mathrm{~J})$. As could be seen in Figure 3I and 3J, a marked emission intensity was observed for the palbociclib-treated tumours treated with HeckGal probe, and senescent cells could be visualized up to a depth of $150 \mu \mathrm{m}$. These results clearly indicated the ability of HeckGal probe for tracking $\beta$-Gal activity at different depths using two-photon microscopy.

To assess the versatility of the probe, HeckGal was also tested to detect cellular senescence in a renal fibrosis model. For this purpose, C57BL/6J male mice were i.p. injected with a single dose of $250 \mathrm{mg} / \mathrm{kg}$ of FA in order to generate renal fibrosis. 34 days postFA injection, the presence of cellular senescence in the kidneys was evaluated with p21 IHC immunostaining. An increase in p21 signal was observed in the kidneys of FA treated-mice, confirming cellular senescence (Figure 4A). Figure 4C shows the quantification of positive p21 nuclei. Once cellular senescence was assessed in this model, $200 \mu \mathrm{l}$ of HeckGal were i.p. injected at a concentration of $6.6 \mathrm{mg} / \mathrm{ml}$ (DMSO 1\%-Corn oil).
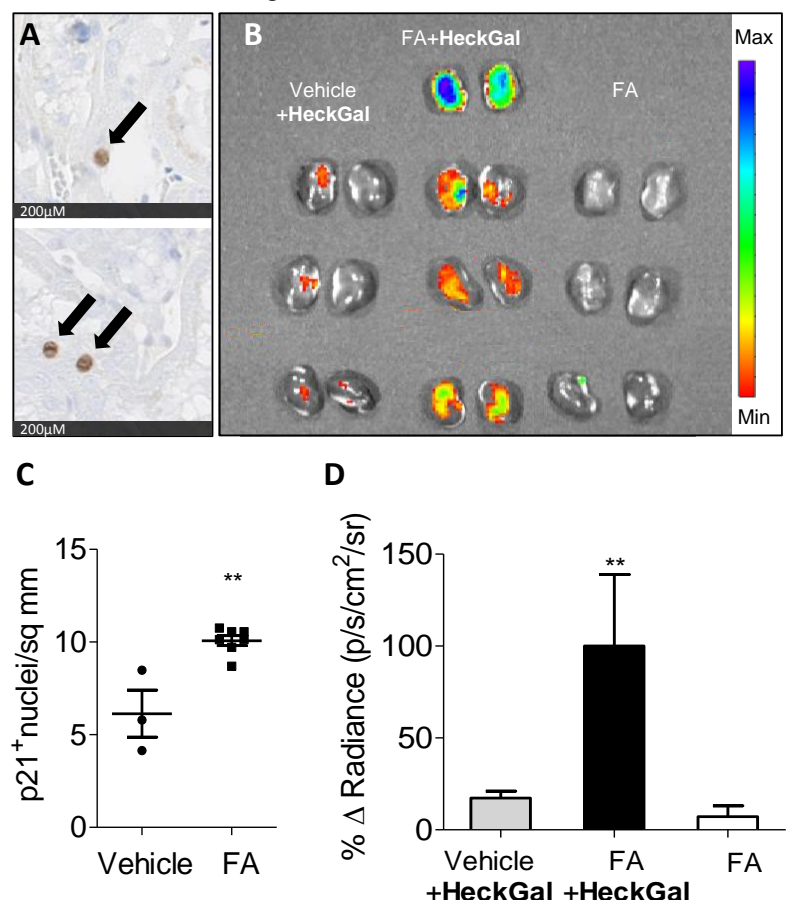

Figure 4. (A) Immunostaining for p21 in kidney slides. (B) IVIS images of kidneys from mice with renal fibrosis induced by FA treatment. From left to right: Vehicle mice + HeckGal $(6 \mathrm{mg} / \mathrm{ml} 200 \mu \mathrm{l})$; FA-treated mice (with renal fibrosis) + HeckGal $(6.6 \mathrm{mg} / \mathrm{ml} 200 \mu \mathrm{l})$; and FA-treated mice (with renal fibrosis. Mice were sacrificed five hours post-HeckGal injection. (C) Quantification of p21 signal in paraffin sections of kidney from vehicle and FA-treated mice. Error bars represent SEM. (D) Quantification of average radiance intensity from kidneys showed in 3B image. Error bars represent SEM ( $\mathrm{n}=3$ for control mice treated with probe and FAtreated mice and $\mathrm{n}=4$ for FA-treated mice+HeckGal). 
Mice were euthanized 5 hours post-HeckGal treatment, kidneys were excised and analyzed by IVIS imaging. Kidneys from control mice treated with HeckGal presented a very weak fluorescent signal (Figure 4B left), whereas kidneys from FA-treated mice and injected with HeckGal (Figure 4B middle) exhibited an intense emission (5.8-fold higher). FA-treated mice did not present any significant auto-fluorescence in the absence of the HeckGal probe (Figure 4B, right).

\section{CONCLUSION}

In summary, we report herein the synthesis of a new two-photon fluorescent probe for the detection of cellular senescence in vivo. HeckGal is based on a naphthalimide core linked to an acetylated galactose which quenches the emission of Heck fluorophore. HeckGal is hydrolysed into the highly fluorescent Heck fluorophore in presence of the $\beta$-Gal enzyme. In vitro detection of cellular senescence using HeckGal was assessed in senescent SK-Mel103, A549, 4T1, and BJ cell lines, in which senescence was induced by treatment with different therapies. The probe was validated to detect cellular senescence by one-photon and by twophoton confocal images and by FACS. The use of HeckGal to detect cellular senescence was also validated in vivo in BALB/C mice bearing 4T1 breast tumours, where senescence was induced with palbociclib. Ex vivo IVIS images showed that fluorescence ascribed to the hydrolyzed HeckGal probe (Heck fluorophore) was only observed in senescent tumours, whereas a negligible emission was found in other organs. Besides, HeckGal probe was also tested in a renal fibrosis model induced with FA. In this model, emission was only observed in fibrotic senescent kidneys from FA-treated mice. We hope that the studies presented here will help in the field of cellular senescence diagnosis in more translatable in vivo models. We also envisage that HeckGal or similar probes can be essential tools in the detection of senescent cells in aged or damaged tissues, and to assess treatment response of senolytics in aging-related diseases.

\section{ASSOCIATED CONTENT}

Supplemental Information includes chemical characterization of the probe and reaction intermediates, experimental procedures, studies of the mechanism of hydrolysis, studies of fluorescence emission vs. $\mathrm{pH}$, and calculations of quantum yields. Toxicity of HeckGal and Heck in SK-Mel-103 and 4T1 cells is also shown in the supplementary information, as well as some confocal images of SK-Mel-103 with different confocal objectives. The immunohistochemical detection of Ki67 is provided in the supplemental material.

\section{Supplemental Information can be found online at https://}

\section{ACKNOWLEDGMENTS}

R.M laboratory members thank the financial support from the Spanish Government (project RTI2018-100910-B-C41) and the Generalitat Valenciana (project PROMETEO 2018/024). B.L-T. is grateful to the Spanish Ministry of Economy for their $\mathrm{PhD}$ grants (FPU15/02707). I.G. thanks her contract from IDM. J. F.-B thanks to his postdoctoral fellowship (CD19/00038). J.A.L-D. thanks the financial support from the Ministry of Science/MICINN (IJCI-2017-33047). Work in the laboratory of MS was funded by the IRB and by grants from the Spanish Ministry of Economy co-funded by the European Regional Development Fund (ERDF) (SAF2013-48256-R), the European Research Council (ERC-2014-AdG/669622), and the "laCaixa" Foundation. D.M.-E. laboratory is supported by the Cancer Research UK (CRUK) Cambridge Centre Early Detection Programme, by a
CRUK Early Detection OHSU Project Award (C62187/A26989), by a Medical Research Council (MRC) New Investigators Research Grant (NIRG, MR/R000530/1).

\section{AUTHOR CONTRIBUTION}

B. L.-T., J. F. B., and R. M.-M. conceived and designed the research, performed experiments, contributed to the experimental designs, data analysis, discussion and writing. J. F. B. synthesized and characterized all organic molecules with the help of B. L.-T. J. F. B. performed HPLC and UV studies. B. L.-T., carried out in vitro studies with SK-Mel-103 and 4T1 cell lines. J.A. L.-D. performed flow cytometry experiments. M. P.-R., E. G.-G. and D.M-E. performed the experiments with human lung carcinoma and contributed to the discussion of the manuscript. B. L.-T. and I. G. carried out in vivo experiments with $4 \mathrm{~T} 1$ breast cancer model. M.S. provided expertise on cellular senescence, contributed to the discussion and designed the in vivo experiment with renal fibrosis model, which was accomplished by J.A. L.-D., M. R and B.L.-T. B. L.-T., J. F. B., F.S.-G. and R. M.-M. analyzed the data. B. L.-T., J. F. B., F. S and R. M.-M. wrote the manuscript with feedback from all the authors.

\section{REFERENCES}

(1) Munoz-Espin, D., and Serrano, M. (). Cellular senescence: from physiology to pathology. Nat. Rev. Mol. Cell. Biol. 2014, 15, 482-496.

(2) Hayflick, L., and Moorhead, P. S. . The serial cultivation of human diploid cell strains. Exp. Cell Res. 1961, 25, 585-621.

(3) Bielak-Zmijewska, A., Mosieniak, G., and Sikora, E. Is DNA damage indispensable for stress-induced senescence?. Mech. Ageing Dev. 2018, 170, 13-21.

(4) Mosteiro, L., Pantoja, C., de Martino, A., and Serrano, M. Senescence promoted in vivo reprogramming through p16INK4a and IL-6. Aging Cell 2018, 17, e12711.

(5) Ritschka, B., Storer, M.; Mas, A., Heinzmann, F., Ortells, M. C., Morton, J. P., Sansom, O. J., Zender, L., and Keyes, W. M. The senescence-associated secretory phenotype induces cellular plasticity and tissue regeneration. Genes Dev. 2017, 31, 172-183.

(6) McHugh, D., and Gil, J. Senescence and aging: Causes, consequences, and therapeutic avenues. J. Cell Biol. 2018, 217, 6577.

(7) Baker, D. J., Childs, B. G., Durik, M., Wijers, M. E., Sieben, C., J., Zhong, J., Saltness, R. A., Jeganathan, K. B., Verzosa, G. C., Pezeshki, A., Khazaie, K., Miller, J. D., and van Deursen, J. M. Naturally occurring p16INK4a-positive cells shorten healthy lifespan. Nature 2016, 530, 184-189.

(8) Baker, D. J., Wijshake, T., Tchkonia, T., LeBrasseur, N. K., Childs, B. G., van de Sluis, B., Kirkland, J. L., and van Deursen, J. M. Clearance of p16INK4a-positive senescent cells delays ageing-associated disorders. Nature 2011, 479, 232-236.

(9) a) Soto-Gamez, A., and Demaria, M. Therapeutic interventions for aging: The case of cellular senescence. Drug Discov. Today 2017, 22, 786-795. b) González- Gualda, E., Pàez- Ribes, M., Lozano- Torres, B., Macias, D., Wilson III, J. R., GonzálezLópez, C., Ou, H.- L., Mirón- Barroso, S., Zhang, Z., LéridaViso, A. Blandez, J. F., Bernardos, A., Sancenón, F., Rovira, M., Fruk, L., Martins, C. P., Serrano, M., Doherty, G. J., MartínezMáñez, R., Muñoz- Espín, D. Galacto- conjugation of Navitoclax as an efficient strategy to increase senolytic specificity and reduce platelet toxicity. Aging Cell. 2020, 19(4), e13142. c) Galiana, I., LozanoTorres, B., Sancho, M., Alfonso, M., Bernardos, A., Bisbal, V., Serrano, M., Martínez-Máñez, R., Orzáez, M. Preclinical antitumor efficacy of senescence-inducing chemotherapy combined with a nanoSenolytic. 2020, 323, 624-634. 
(10) a) Lozano-Torres, B., Estepa-Fernández, A., Rovira, M., Orzáez, M., Serrano, M., Martínez-Máñez, R., and Sancenón, F. The Chemistry of Senescence. Nat. Rev. Chem. 2019, 3, 426-441. b) Lozano- Torres, B., Blandez, J. F., Galiana, I., GarcíaFernández, A., Alfonso, M. Marcos, M. D., Orzáez, M. Sancenón, F., Martínez- Máñez, R. Real- Time In Vivo Detection of Cellular Senescence through the Controlled Release of the NIR Fluorescent Dye Nile Blue. Angew. Chem. Int. Ed. 2020, 59(35), 15152-15156.

(11) Paez-Ribes, M., González-Gualda, E., Doherty, G. J., and Muñoz-Espín, D. Targeting senescent cells in translational medicine. EMBO Mol. Med. 2019, 11, e10234

(12) Funayama, R., and Ishikawa, F. Cellular senescence and chromatin structure. Chromosoma 2007, 116, 431-440.

(13) Zhang, R., and Adams, P. D.. Heterochromatin and its relationship to cell senescence and cancer therapy. Cell Cycle 2007, 6, 784-789.

(14) Serrano, M., Hannon, G. J., and Beach, D. A. New regulatory motif in cell-cycle control causing specific inhibition of cyclin D/CDK4. Nature 1993, 366, 704-707.

(15) Serrano, M., Lin, A. W., McCurrach, M. E., Beach, D., and Lowe, S. W. Oncogenic Ras provokes premature cell senescence associated with accumulation of p53 and p16INK4a. Cell 1997, $88,593-602$.

(16) Sharpless, N. E., and Sherr, C. J. Forging a signature of in vivo senescence. Nat. Rev. Cancer 2015, 15, 397-408.

(17) Hernandez-Segura, A., Nehme, J., and Demaria, M. Hallmarks of cellular senescence. Trends Cell Biol. 2018, 28, 436453

(18) Childs, B. G., Baker, D. J., Kirkland, J. L., Campisi, J., and van Deursen, J. M. Senescence and apoptosis: dueling or complementary cell fates?. EMBO Rep. 2014, 15, 1139-1153.

(19) Takahashi, A., Ohtani, N., and Hara, E. Irreversibility of cellular senescence: dual roles of p16INK4a/ $\mathrm{Rb}$-pathway in cell cycle control. Cell Div. 2007, 2, 10-15.

(20) Guerrero, A., and Gil, J. HMGB2 holds the key to the senescence-associated secretory phenotype. J. Cell Biol. 2016, 215, 297-299.

(21) Krtolica, A., Parrinello, S., Lockett, S., Desprez, P. Y., and Campisi, J. Senescent fibroblasts promote epithelial cell growth and tumorigenesis: A link between cancer and aging. Proc. Natl. Acad. Sci. U.S.A. 2001, 98, 12072-12077.

(22) Dimri, G. P., Lee, X., Basile, G., Acosta, M., Scott, G., Roskelley, C., Medrano, E. E., Linskens, M., Rubelj, I., and PereiraSmith, O. A biomarker that identifies senescent human cells in culture and in aging skin in vivo. Proc. Natl. Acad. Sci. U.S.A. 1995, 92, 9363-9367.

(23) Rotman, B. Measurement of activity of single molecules of beta-D-galactosidase. Proc. Natl. Acad. Sci. U.S.A. 1961, 47, 1981-1991.

(24) Rotman, B., Zderic, J. A., and Edelstein, M. Fluorogenic substrates for beta-D-galactosidases and phosphatases derived from fluorescein (3,6-dihydroxyfluoran) and its monomethylether. Proc. Natl. Acad. Sci. U.S.A. 1963, 50,1-6.

(25) Strachan, R., Wood, J., and Hirschmann, R. Synthesis and properties of 4-methyl-2-oxo-1,2-benzopyran-7-yl $\beta$-Dgalactoside (galactoside of 4-methylumbelliferone). J. Org. Chem. 1962, 27, 1074-1075.

(26) Horwitz, J. P., Chua, J., Curby, R. J., Tomson, A. J., Da Rooge, M. A., Fisher, B. E., Mauricio, J., and Klundt, I. Substrates for cytochemical demonstration of enzyme activity. I. Some substituted 3-indolyl- $\beta$-D-glycopyranosides. J. Med. Chem. 1964, 7, 574-575.

(27) Zhang, J., Li, C., Dutta, C., Fang, M., Zhang, S., Tiwari, A., Werner, T., Luo, F. T., and Liu, H. A novel near-infrared fluorescent probe for sensitive detection of $\beta$-galactosidase in living cells. Anal. Chim. Acta 2017, 968, 97-104.
(28) Dai, Z. R., Ge, G. B., Feng, L., Ning, J., Hu, L. H., Jin, Q., Wang, D. D., Lv, X., Dou, T. Y., Cui, J. N., and Yang, L. A highly selective ratiometric two-photon fluorescent probe for human cytochrome P450 1A. J. Am. Chem. Soc. 2015, 137, 14488-14495. (29) Zhou, L., Zhang, X., Wang, Q., Lv, Y., Mao, G., Luo, A., Wu, Y., Wu, Y., Zhang, J., and Tan, W. Molecular engineering of a TBET-based two-photon fluorescent probe for ratiometric imaging of living cells and tissues. J. Am. Chem. Soc. 2014, 136, 9838 9841

(30) Cepraga, C., Gallavardin, T., Marotte, S., Lanoë, P. H., Mulatier, J. C., Lerouge, F., Parola, S., Lindgren, M., Baldeck, P. L., Marvel, J., Maury, O., Monnereau, C., Favier, A., Andraud, C., Leverrier, Y., and Charreyre, M. T. Biocompatible well-defined chromophore-polymer conjugates for photodynamic therapy and two-photon imaging. Polym. Chem. 2013, 4, 61-67.

(31) Kamiya, M., Kobayashi, H., Hama, Y., Koyama, Y., Bernardo, M., Nagano, T., Choyke, P. L., and Urano, Y. An enzymatically activated fluorescence probe for targeted tumor imaging. $J$. Am. Chem. Soc. 2007, 129, 3918-3929.

(32) Gu, K., Xu, Y., Li, H., Guo, Z., Zhu, S., Zhu, S., Shi, P., James T. D., Tian, H., and Zhu, W. H. Real-time tracking and in vivo visualization of $\beta$-galactosidase activity in colorectal tumor with a ratiometric near-infrared fluorescent probe. J. Am. Chem. Soc. 2016, 138, 5334-5340.

(33) Oushiki, D., Kojima, H., Takahashi, Y., Komatsu, T., Terai, T., Hanaoka, K., Nishikawa, M., Takakura, Y., and Nagano, T. Near-infrared fluorescence probes for enzymes based on binding affinity modulation of squarylium dye scaffold. Anal. Chem. 2012, 84, 4404-4410.

(34) Tung, C. H., Zeug, Q., Shah, K., Kim, D. E., Schellingerhout, D., and Weissleder, R. In vivo imaging of $\beta$-galactosidase activity using far red fluorescent switch. Cancer Res. 2004, 64, 15791583.

(35) Urano, Y., Kamiya, M., Kanda, K., Ueno, T., Hirose, K., and Nagano, T. Evolution of fluorescein as a platform for finely tunable fluorescent probes. J. Am. Chem. Soc. 2005, 127, 4888-4894.

(36) Egawa, T., Koide, Y., Hanaoka, K., Komatsu, T., Terai, T., and Nagano, T. Development of a fluorescein analogue, TokyoMagenta, as a novel scaffold for fluorescence probes in red region. Chem. Commun. 2011, 47, 4162-4164.

(37) Kamiya, M., Asanuma, D., Kuranaga, E., Takeishi, A., Sakabe, M., Miura, M., Nagano, T., and Urano, Y. $\beta$-Galactosidase fluorescence probe with improved cellular accumulation based on a spirocyclized rhodol scaffold. J. Am. Chem. Soc. 2011, 133, 12960-12963.

(38) Sakabe, M., Asanuma, D., Kamiya, M., Iwatate, R. J., Hanaoka, K., Terai, T., Nagano, T., and Urano, Y. Rational design of highly sensitive fluorescence probes for protease and glycosidase based on precisely controlled spirocyclization. J. Am. Chem. Soc. 2013, 135, 409-414.

(39) Peng, L., Gao, M., Cai, X., Zhang, R., Li, K., Feng, G., Tong, A., and Liu, B. A fluorescent light-up probe based on AIE and ESPIT processes for $\beta$-galactosidase activity detection and visualization in living cells. J. Mater. Chem. B 2015, 3, 9168-9172.

(40) Han, J., Han, M. S., and Tung, C. H. A fluorogenic probe for $\beta$-galactosidase activity imaging in living cells. Mol. BioSyst. 2013, 9, 3001-3008.

(41) Zhang, X. X., Wu, H., Li, P., Qu, Z. J., Tan, M. Q., and Han K. L. A versatile two-photon fluorescent probe for ratiometric imaging E. coli $\beta$-galactosidase in live cells and in vivo. Chem. Commun. 2016, 52, 8283-8286.

(42) Lee, H. W., Heo, C. H., Sen, D., Byun, H. O., Kwak, I. H., Yoon, G., and Kim, H. M. Ratiometric two-photon fluorescent probe for quantitative detection of $\beta$-Galactosidase activity in senescent cells. Anal. Chem. 2014, 86, 10001-10005.

(43) Lozano-Torres, B., Galiana, I., Rovira, M., Garrido, E., Chaib, S., Bernardos, A., Muñoz-Espín, D., Serrano, M., Mar- 
tínez-Máñez, R., and Sancenón, F. An OFF-ON two-photon fluorescent probe for tracking senescence in vivo. J. Am. Chem. Soc. 2017, 139, 8808-8811.

(44) de la Torre, C., Toscani, A., Marín-Hernández, C., Robson, J. A., Terencio, M. C., White, A. J. P., Alcaraz, M. J., Wilton-Ely, J. D. E. T., Matínez-Máñez, R., and Sancenón, F. Ex vivo tracking of endogenous $\mathrm{CO}$ with a ruthenium(II) complex. J. Am. Chem. Soc. 2017, 139, 18484-18487.

(45) Llopis-Lorente, A., Villalonga, R., Marcos, M. D., MartínezMáñez, R., and Sancenón, F. A versatile new paradigm for the design of optical nanosensors based on enzyme-mediated detachment of labeled reporters: The example of urea detection. Chem. Eur. J. 2019, 25, 3575-3581.

(46) Oroval, M., Coll, C., Bernardos, A., Marcos, M. D., Martínez-Máñez, R., Shchukin, D., and Sancenón, F. Selective fluorogenic sensing of As(III) using aptamer-capped nanomaterials. ACS Appl. Mater. Interfaces 2017, 9, 11332-11336.

(47) Lozano-Torres, B., Pascual, Ll., Bernardos, A., Marcos, M. D., Jeppesen, J. O., Salinas, Y., Martínez-Máñez, R., and Sancenón, F. Pseudorotaxane capped mesoporous silica nanoparticles for 3,4-methylendioxymethanphetamine (MDMA) detection in water. Chem. Commun. 2017, 53, 3559-3562.

(48) Moragues, M. E., Toscani, A., Sancenón, F., MartínezMáñez, R., White, A. J., and Wilton-Ely, J. D. E. T. A chromofluorogenic synthetic "canary" for $\mathrm{CO}$ detection based on a pyrenylvinyl ruthenium(II) complex. J. Am. Chem. Soc. 2014, 136, 11930-11933.

(49) Whittaker, S. R., Mallinger, A., Workman, P., and Clarke, P. A. Inhibitors of cyclin-dependent kinases as cancer therapeutics. Pharmacol. Ther. 2017, 173, 83-105. 